\title{
Consensos em Saúde Bucal aprimorando a Atenção Básica, Ribeirão Preto, SP
}

\author{
Guidelines in Oral Health improvement the \\ Primary Health Attention, Ribeirão Preto, SP
}

Kelly M. de Andrade ${ }^{1}$, Stella M. de Andrade ${ }^{2}$, Ana Maria Jabali ${ }^{3}$, Soraya F. Mestriner ${ }^{4}$, Maria do Carmo Gullaci Guimarães Caccia-Bava ${ }^{5}$, Silvana Martins Mishima ${ }^{6}$, Wilson Mestriner $\mathrm{Jr}^{7}$

\begin{abstract}
RESUMO
Atualmente, o desenvolvimento e utilização dos Protocolos clínicos e de regulação na área da saúde, como ferramenta para gestão, têm contribuído para a organização e padronização da prática clínica em diversas áreas assistenciais, bem como conferido clareza quanto aos fluxos a serem percorridos pelos pacientes, em busca do cuidado à saúde. Por meio de estudo de abordagem qualitativa e valendo-se da Técnica Delphi na busca da convergência de opiniões, objetivou-se obter um consenso entre os participantes da pesquisa para a adoção dos conceitos que pudessem subsidiar a criação e implantação de um Protocolo em Saúde Bucal a ser aplicado na Rede Pública de Saúde no município de Ribeirão Preto. A amostra inicial de caráter aleatório, a partir de sorteio entre as Unidades de Saúde do Distrito Oeste de Ribeirão Preto foi composta por 13 dentistas e profissionais da equipe de saúde sendo que a metodologia foi desenvolvida em 3 etapas. A primeira iniciou-se com o grupo de dentistas das unidades sorteadas, que responderam a uma série de questões sendo os resultados dessa primeira fase compilados e a síntese das tarefas reenviada aos membros para que respondessem novamente, reconsiderando as suas respostas. O produto final foi discutido em Oficina composta pelos dentistas entrevistados, representantes das equipes de saúde, da Secretaria de Saúde e dos usuários do serviço a fim de que o instrumento pudesse ser construído contemplando o ponto de vista de todos os envolvidos no processo. Resultados: $O$ trabalho identificou concordância final entre os participantes, pelo que se comprovou que a Técnica Delphi favoreceu a tomada de decisões dos profissionais e usuários acerca da consolidação de conceitos próprios da atenção básica em saúde bucal para o desenvolvimento dos cuidados nessa área, colaborando potencialmente para as decisões racionais e aprimoramento da qualidade da assistência. Mostrou-se uma ferramenta com potência para a busca de consensos na área de Saúde Pública.
\end{abstract}

Palavras-chaves: Protocolos Clínicos. Atenção Básica. Saúde bucal.

1. Mestre, Departamento de Odontologia Restauradora da Faculdade de Odontologia de Ribeirão Preto da Universidade de São Paulo, Ribeirão Preto, São Paulo, Brasil (FORP-USP)

2. Cirurgiã-dentista residente do curso de Residência Multiprofissional em Saúde da Família e Comunidade do Departamento de Medicina da UFSCar, São Carlos, São Paulo, Brasil.

3. Cirurgiã Dentista, Departamento de Auditoria e Controle da Secretaria Municipal de Saúde de Ribeirão Preto

4. Docente, Departamento de Clínica Infantil, Odontologia Preventiva e Social da FORP-USP

5. Docente, Departamento de Medicina Social da FMRP-USP

6. Docente, Departamento Materno Infantil e Saúde Pública da Escola de Enfermagem de Ribeirão Preto da USP

7. Docente, Departamento de Clínica Infantil, Odontologia Preventiva e Social da FORP-USP
Correspondência Prof. Dr. Wilson Mestriner Junior Avenida do Café, s/n, Bairro Monte Alegre 14040-904 - Ribeirão Preto - SP

(16)3602-4142 mestri@ forp.usp.br

Artigo recebido em 04/04/2011 Aprovado para publicação em 17/11/2011 


\section{Introdução}

A atenção básica à saúde constitui o eixo de organização do sistema de saúde, e apresenta potência para transformar a lógica do modelo de atenção vigente. ${ }^{1}$ De acordo com inúmeras pesquisas, a atenção básica, funcionando adequadamente, de forma resolutiva, oportuna e humanizada, é capaz de resolver, com qualidade, cerca de $85 \%$ dos problemas de saúde da população. ${ }^{2}$

Entretanto, uma das grandes preocupações que permeiam a Atenção Básica, não só na área da Odontologia como também nas outras áreas da saúde, é o fluxo inadequado no que tange a referência e contrareferência dos usuários no sistema de saúde tendo por conseqüência a demora do paciente em conseguir o tratamento especializado. De acordo com os complexos reguladores, tal fato se deve, provavelmente, pelos encaminhamentos serem feitos de maneira incorreta e também pelos pacientes serem referenciados ao nível secundário e terciário sem terem obtido o tratamento básico completado, não estando aptos, portanto, para a realização do tratamento especializado. ${ }^{3}$

Esforços para a organização do sistema de saúde bucal com vistas à obtenção do atendimento integral aos pacientes em todos os níveis de atenção à saúde - princípio ético do SUS, poderiam ser favorecidos com a construção de Protocolos Clínicos e de Regulação abrangentes, que incluíssem todos os procedimentos clínicos Odontológicos a serem realizados na Atenção Básica para uma correta adequação e encaminhamento do paciente para o Centro de Especialidades Odontológicas. ${ }^{4}$

À despeito de sua importância, os protocolos clínicos e de regulação devem contar com a valorização e a adesão dos profissionais da Rede Pública, sob o risco dele ser negado por todos. Considera-se que o exercício da co-gestão em saúde deve partir da percepção dos profissionais de saúde que lidam cotidianamente com situações adversas e são os responsáveis pela realização de um diagnóstico global e identificação das necessidades prioritárias de tratamento em conjunto com os pacientes, o que estimula a autonomia e co-responsabilização de todos os que fazem parte do processo de produção do cuidado em saúde.

Deste modo, com o intuito de reverter a forma cindida com que a prática Odontológica tem sido tratada no âmbito do SUS em relação aos demais serviços de saúde, idealizou-se a participação de todos os profissionais membros das equipes de saúde e dos usuários no processo de criação de um consenso para a área de saúde bucal a partir da Atenção Básica. Para a obtenção de um nível de concordância razoável, conforme apontado na literatura, o recurso da Técnica Delphi, vem sendo utilizada visando estabelecer o grau de aceitação e concordância requerida para efetivação de ações na área da saúde. ${ }^{5}$

Dessa forma, a atual investigação tem por objetivo testar a técnica Delphi como recurso para a elaboração de consensos na área Odontológica de Saúde Pública visando contribuir para o processo de construção e validação de um Protocolo Clínico e de Regulação Odontológico a ser implementado na Atenção Básica do município de Ribeirão Preto.

\section{Material e métodos}

Aspectos éticos: O estudo foi realizado após a aprovação do Comitê de Ética em Pesquisa da Faculdade de Odontologia de Ribeirão Preto da Universidade de São Paulo (Processo ${ }^{\circ}$ 2007.1.1106.58.7.) e a assinatura dos Termos de Consentimento Livre e Esclarecido.

\section{Contexto do estudo}

A rede básica do município de Ribeirão Preto é constituída por 30 Unidades Básicas de Saúde, cinco Unidades Básicas e Distritais de Saúde e 18 Equipes de Saúde da Família, sendo que destas 11 são distribuídas no Distrito Oeste, uma no Distrito Central e seis no Distrito Norte. Essa estrutura permite o atendimento nos níveis primários e especializado à saúde de aproximadamente 140 mil habitantes. ${ }^{6}$

Esta pesquisa teve como campo o Distrito Oeste do Município de Ribeirão Preto, o Distrito de atuação da Universidade de São Paulo, responsabilidade definida a partir de um convênio estabelecido entre a Prefeitura Municipal de Ribeirão Preto e a Universidade de São Paulo (USP), onde as várias Unidades de Ensino do Campus da USP assumem responsabilidades pela coordenação das atividades assistenciais, de ensino e pesquisa de acordo com as diretrizes emanadas da Secretaria Municipal de Saúde de Ribeirão Preto e que conta com unidades de saúde tradicional (UBS), Unidades de Agentes Comunitários de Saúde (UACS) e de saúde da família (ESF).

Com a participação de profissionais lotados nas unidades de saúde da rede básica com a configuração acima foi constituída uma amostra de caráter aleatório, a partir de sorteio. Foram selecionados, como su- 
jeitos da pesquisa, para a primeira e segunda etapa desta pesquisa, todos os cirurgiões dentistas das unidades selecionadas, segundo a Tabela 1 , correspondendo o equivalente a 13 cirurgiões dentistas participantes. Para a terceira etapa (etapa grupal) os demais profissionais da equipe, os representantes dos usuários de cada unidade e os representantes da divisão odontológica foram convidados a participar, constituindo uma amostra de 20 pessoas.

\section{Coleta de dados}

Dentre as características da técnica Delphi, ressalta-se o respeito e a valorização da experiência e do conhecimento de cada um dos participantes que, de uma forma dirigida, colocam à apreciação coletiva seus julgamentos subjetivos, mas resultantes de um longo processo de sistematização do conhecimento adquirido na prática. ${ }^{7} \mathrm{De}$ forma geral, a Técnica Delphi consulta um grupo de especialistas a respeito de eventos futuro, através de um questionário repassado continuadas vezes, até a obtenção de uma convergência das respostas, um consenso, que representa a consolidação do julgamento intuitivo do grupo. ${ }^{8}$

Pressupõe-se que o julgamento coletivo, em um processo grupal, ao ser bem organizado, é melhor do que a opinião de um só indivíduo. $\mathrm{O}$ anonimato dos respondentes, a distribuição dos resultados e o feedback de respostas do grupo para reavaliação nas rodadas subseqüentes são as principais características deste método.

Durante a busca pelo consenso sobre os itens que seriam contemplados pelo protocolo, a Técnica Delphi apresentou grande flexibilidade, uma vez que relativizou fatores interpessoais que frequientemente influenciam os grupos ou comitês de consenso quando os participantes estão frente a frente. Em sua pri- meira etapa foi utilizado um questionário individual garantindo-se o anonimato dos respondentes.

À semelhança do estudo de Gontijo, 2007, ${ }^{9}$ utilizou-se a técnica Delphi modificada, onde se alterou o julgamento dos pleitos pela apreciação da equipe de saúde da Unidade selecionada, uma vez que foi considerada a prática de quem está executando o trabalho, ou seja, aqueles que estão inseridos no processo de trabalho na saúde bucal.

\section{Etapas da pesquisa}

O estudo foi realizado em três etapas: a primeira necessária para se iniciar a busca pelos conteúdos que deveriam ser contemplados pelo Protocolo; a segunda, na qual foram repassados os itens respondidos na primeira etapa para que houvesse reconsideração das respostas que se sobrepunham ou apresentavam convergência a um conceito ou tema; e a terceira, na qual as respostas da segunda etapa foram aglutinadas coerentemente, segundo as diretrizes presentes no $\mathrm{Ca}$ derno da Atenção Básica no 17: Saúde Bucal 2006, ${ }^{1}$ na forma de categorias e discutidas numa Oficina entre todo o grupo.

\section{Detalhamento}

Primeira Etapa: realizou-se um primeiro contato pessoal com os sujeitos da pesquisa, para informação e esclarecimento sobre as atividades a serem desenvolvidas e obtenção do Termo de Consentimento Livre e Esclarecido. Com a presença e colaboração de profissionais gestores da secretaria de saúde, foi explicada aos profissionais a necessidade e a estratégia para se chegar a um consenso dos itens adequados para a criação ou não, de um protocolo clinico e de regulação a ser implementado pela Secretaria Municipal de Saúde a fim de se organizar o sistema e

\section{Tabela 1}

Caracterização das unidades de saúde selecionadas para o estudo e número de cirurgiões dentista, Distrito Oeste, Ribeirão Preto-SP

\begin{tabular}{lcc}
\hline Unidades de Saúde & Tipo & Dentistas participantes \\
\hline UBS A & Unidade Tradicional & 4 \\
UBS B & Unidade Tradicional & 3 \\
UBSC & Unidade Mista & 3 \\
Núcleo de Saúde da Família A & PSF & 1 \\
Núcleo de Saúde da Família B & PSF & 1 \\
Núcleo de Saúde da Família C & PSF & 1 \\
\hline
\end{tabular}


o fluxo de encaminhamentos odontológicos para os serviços especializados.

Nesta etapa participaram todos os dentistas das unidades escolhidas, 13 dentistas das seis Unidades de Saúde. Todos receberam um envelope da mesma cor e tamanho, com o objetivo de manter o anonimato sendo que o envelope não foi identificado.

Cada envelope continha uma carta de esclarecimento sobre os objetivos da pesquisa e uma Lista com quatro questões indicadas no Quadro I, que deveriam ser respondidas em uma folha de papel almaço, também fornecida pelos pesquisadores.

\section{Quadro I}

Lista de questões para a Primeira Etapa

1- De que forma um protocolo em saúde bucal ajudaria organizar o processo de trabalho em sua equipe?

2- Qual a contribuição que a criação de um Protocolo Clínico e de Regulação daria no seu processo de trabalho?

3- Quais os conteúdos que o Protocolo em saúde bucal, caso seja criado, deveria contemplar?

4- Quais as situações do cotidiano deveriam ser previstas num Protocolo Clínico?

Foi solicitado aos participantes que citassem três sugestões sobre cada uma das perguntas para que houvesse maior quantidade e diversidade de sugestões.

A finalidade foi elaborar uma listagem inicial que serviu de subsídio para iniciar a busca de opiniões entre os cirurgiões-dentistas sobre as sugestões para o protocolo.

Após as respostas serem coletadas, coube aos pesquisadores compilar uma nova lista com todas as respostas obtidas na etapa anterior (mantendo-se a fidelidade das respostas). Essas respostas seriam repassadas aos cirurgiões dentistas participantes a fim de que pudessem reconsiderar a sua opinião nesta segunda etapa.

Como na primeira etapa foi pedido aos participantes que dessem três sugestões para cada uma das quatro perguntas, o esperado para essa nova lista era que houvesse $n=39$ sugestões dadas pelos cirurgiões dentistas para cada uma das perguntas da primeira etapa. Todavia, houve uma diferença no número de sugestões dadas. Essa diferença se deve à liberdade da técnica, que permite aos sujeitos da pesquisa que citem sugestões ora a menos, ora a mais do que o esperado. Assim para a primeira questão obtive-se 27 respostas, segunda 32, terceira 53 e finalmente, 37 para quarta e última pergunta.

$\mathrm{Na}$ segunda etapa, os cirurgiões dentistas participantes receberam a lista de sugestões compilada pelos pesquisadores a partir das respostas da primeira etapa. (Quadro II)

Com a lista de respostas em mãos, os profissionais, desenvolveram a leitura e reconsideração de suas respostas. Cada profissional deveria selecionar as cinco respostas que contivessem os itens mais adequados para conter no protocolo. Da mesma forma, os profissionais responderam anonimamente.

Após análise das listas coletadas da segunda etapa (Quadro III) os pesquisadores criaram categorias que representassem os conteúdos essenciais das respostas, ou que tivessem seu conteúdo fundamentado em documentos já existentes preconizados pelo Sistema Único de Saúde. (Quadro IV)

Ressalta-se que os participantes só tiveram contato com as categorias na Terceira Etapa, na qual foi realizada uma Oficina de Discussão com diferentes membros das equipes.

Na terceira e última etapa participaram da oficina além dos cirurgiões dentistas, os profissionais membros da equipe, sendo importante salientar a participação dos profissionais membros da Divisão Odontológica da Secretaria Municipal de Saúde e representantes dos usuários. Foi neste momento que os participantes entraram em contato com o elenco de indicações feitas pelos cirurgiões-dentistas na primeira e segunda etapa. Após analisarem os conteúdos das categorias de forma individual, os participantes se reuniram em grupos e puderam novamente reavaliar suas posições e ouvir as justificativas dos colegas (etapa grupal).

Os grupos foram formados de forma heterogênea, privilegiando a presença de todas as áreas em cada grupo, conforme a disposição da Tabela 2.

Nos grupos foi solicitado que se discutisse cada categoria e após determinado tempo foi estabelecido o nível de concordância segundo a escala Likert, com cinco níveis, como segue: I- Discordo totalmente; IIDiscordo parcialmente; III- Não tenho opinião; IVConcordo parcialmente; V Concordo totalmente. Após as discussões, cada grupo chegou a um consenso pertinente as categorias apresentadas. De forma organizada, cada grupo: G1,G2 e G3 foi expondo para os presentes o nível de concordância do grupo.(Tabela 3). 


\section{Quadro II}

Exemplos de respostas da primeira etapa repassadas através da Lista da Segunda Etapa

\begin{tabular}{|c|c|}
\hline Questão & Exemplo de Respostas contidas na Lista da segunda etapa \\
\hline 1 & $\begin{array}{l}\text { - ( ) rapidez no encaminhamento(fluxograma) para as especialidades } \\
\text { - ( ) uniformizar o trabalho da equipe } \\
\text { - ( ) facilitar o trabalho da equipe } \\
\text { - ( ) gadronização no atendimento } \\
\text { ( ) garantir a avaliação do processo de trabalho }\end{array}$ \\
\hline 2 & $\begin{array}{l}\text { - ( ) segurança e agilidade nas condutas } \\
\text { - ( ) visualização global de todo o sistema } \\
\text { - ( ) instrumento importante frente a problemas de ordem jurídica, ou seja, um respaldo para os profissionais. } \\
\text { - ( ) será um documento, no qual o cirurgião dentista poderá mostrar aos pacientes, quando estes por } \\
\text { ventura, não aceitarem a conduta estabelecida pelo dentista. }\end{array}$ \\
\hline 3 & $\begin{array}{l}\text { - ( ) acolhimento } \\
\text { - ( ) emergências } \\
\text { - ( ) agendamento } \\
\text { - ( ) atençãáo básica - classificação de risco; procedimentos clínicos; referenciamento } \\
\text { - ( ) estimulo ao PSF (Estratégia) } \\
\text { - ( ) abordar riscos para carie dentaria, doença periodontal, câncer bucal, DTM, relacionando-os a outros } \\
\quad \text { agravos de saúde (risco comuns). }\end{array}$ \\
\hline 4 & $\begin{array}{l}\text { - ( ) hemorragias } \\
\text { - ( ) prevenção, flúor e orientação de escovação } \\
\text { - ( ) qual deve ser a prioridade de atendimento } \\
\text { - ( ) promoçoção da saúde bucal e da saúde } \\
\text { - ( ) atendimento à gestante } \\
\text { - ( ) atendimento à paciente com comprometimentos sistêmicos: diabetes, hipertensão } \\
\text { - ( ) traumas }\end{array}$ \\
\hline
\end{tabular}

\section{Quadro III}

Respostas literais presentes na segunda etapa e frequiência

\begin{tabular}{|c|c|c|}
\hline Questão & & $\begin{array}{l}\text { Número de vezes que } \\
\text { a resposta foi escolhida }\end{array}$ \\
\hline \multirow{9}{*}{1} & - uniformizar o trabalho da equipe & 4 \\
\hline & - facilitar o trabalho da equipe & 4 \\
\hline & - definir o fluxo de atenção em saúde bucal & 8 \\
\hline & - colaborará com a intersetorialização entre os profissionais de saúde & 5 \\
\hline & $\begin{array}{l}\text { - contemplar critérios de risco comuns de agravos a saúde geral e a saúde } \\
\text { bucal pode potencializar as propostas de seguimento de usuários e/auxiliar, } \\
\text { melhorando as atividades de prevenção e promoção }\end{array}$ & 6 \\
\hline & - desencadear motivação nas equipes para discussão sobre saúde bucal & 5 \\
\hline & - organização do trabalho & 4 \\
\hline & - permitir a visibilidade da Odontologia para a equipe e usuário & 2 \\
\hline & - dividir responsabilidades & 2 \\
\hline
\end{tabular}


2 - agilizar o trabalho da equipe 3

- direcionar o fluxo de encaminhamentos 5

- segurança e agilidade nas condutas 2

- adequação do atendimento com a real condição do trabalho do profissional (material, estrutura ou pessoal)

- organizar o processo de trabalho, uniformização e hierarquização das ações e condutas, com o objetivo de nos aproximarmos dos princípios do SUS

- garantir a avaliação do processo de trabalho e dos seus resultados aos profissionais, gerar dados para o planejamento das ações em saúde bucal 3

- guiar o cirurgião dentista, resolver certos problemas com mais agilidade 3

- será importante por ser um aparato frente à problemas de ordem jurídicas, ou seja, um respaldo para os profissionais.

- facilitar a comunicação entre profissionais que atendem o mesmo paciente e melhorar e/ou assegurar a qualidade da atenção em saúde bucal.

- postura ética ,visando indicar condutas mais adequadas para cada situação encontrada e assegurar medidas para o controle e prevenção dos agravos 6

- conhecer o SUS e acreditar na sua proposta 3

- promover uma transparência na parceria SMS, prestadores e universidades) 3

- esclarecer população/trabalho da saúde e prestadores, do fluxo a ser seguido para o atendimento odontológico $\quad 5$

- oferecer segurança aos cirurgiões dentistas e pacientes 4

3 - anamnese

- organizar a demanda de pacientes 4

- avaliarmos o risco de cárie de cada paciente 4

- procedimentos clínicos 4

- programar fóruns de debate permanente e com frequência para aperfeiçoar $\mathrm{o}(\mathrm{s}) \operatorname{protocolo}(\mathrm{s}) \quad 2$

- acolhimento 2

- agendamentos preferenciais (bebês, gestantes, diabéticos) 2

- abordar riscos para carie dentaria, doença periodontal, câncer bucal, DTM 2

- situações de urgência e emergência 2

- organização do fluxo de atendimento ( normatização do processo de encaminhamento) especialidades) 4

- prever cursos para equipes 2

- prever condições que otimizem o processo de trabalho e o tempo

- conteúdos relacionados ao fluxo de pacientes ( referência e contra referência) 4

- conteúdos relacionados à terapêutica e farmacoterapia 6

- conteúdos relacionados ao agendamento, seguindo critérios de risco de
saúde bucal, saúde geral.

4 - atendimento à paciente com comprometimentos sistêmicos: diabetes, hipertensão e gestante $\quad 6$

- traumas, hemorragias 3

- atenção básica e seus procedimentos clínicos, prevenção. 5

- atendimento à pacientes especiais e com patologias sistêmicas 6 
- como proceder frente aos pacientes que demoram para receberem tratamento especializado

- protocolo de atendimento aos bebês ( agendamento, idade máxima, procedimentos)

- paciente de urgência e/ou emergência

- situações de urgência e emergência

- farmacoterapia

- organização do fluxo de atendimento ( normatização dos encaminhamentos p/especialidades)

- critério de encaminhamentos para as especialidades 2

- análise dos critérios de risco à saúde bucal 5

- opções de materiais de consumo e terapêuticos 4

- questões relacionadas à biossegurança 4

\section{Quadro IV}

Descrição das categorias criadas a partir dos itens escolhidos na segunda etapa

Nome das categorias Descrição das categorias de acordo com as respostas colhidas

1- Importância do Protocolo

A implantação de Protocolos de Saúde Bucal configura avanço para um novo patamar de organização que contribuirá para o aprimoramento da atenção ofertada à população. Representa o aperfeiçoamento do processo de trabalho da odontologia no âmbito do SUS e contribui para a potencialização de suas ações. A descrição e normatização da dinâmica do atendimento odontológico em todas as suas etapas, contempla os aspectos referentes às diversas faixas etárias atendidas pela rede municipal de saúde bucal, bem como sua organização, constituindo-se em objeto de sua intervenção.O Protocolo é um instrumento de gestão da clínica e tem como propósito à melhoria da qualidade da assistência odontológica, integrando os fluxos e viabilizando o monitoramento do cuidado e intensificando as ações de promoção à saúde bucal.

2-Promoção da saúde

3-Organização do acesso

4-Acolhimento
Aspecto fundamental para efetivação da Atenção Básica é a promoção de saúde, Por ser uma estratégia de articulação transversal que objetiva a melhoria na qualidade de vida e a redução da vulnerabilidade e dos riscos à saúde, possibilitam a população ter melhorias no modo de viver: condições de trabalho, habitação, educação, lazer, cultura, acesso a bens e serviços essenciais. Na odontologia são exemplos ações de fluorterapia, ações de educação em saúde, procedimentos preventivos, atendimento clínico individual nas Unidades de Saúde quando possível.

A ordem de chegada não deve ser o principal critério para o atendimento dos casos, mas a sua gravidade ou o sofrimento do paciente. Neste sentido é que se prioriza o atendimento a qualquer urgência, quando esta estiver ocasionando dor ou sofrimento ao usuário. A urgência, inclusive, é um momento importante para a detecção de indivíduos com maior vulnerabilidade.

O acolhimento é um modo de operar os processos de trabalho em saúde de forma a atender a todos que procuram os serviços de saúde, ouvindo seus pedidos e assumindo no serviço uma postura capaz de acolher, escutar e pactuar respostas mais adequadas aos usuários. Implica prestar um atendimento com resolutividade e responsabilização, orientando, quando for o caso, a população em relação a outros serviços de saúde para a continuidade da assistência e estabelecendo articulações com esses serviços para garantir a eficácia desses encaminhamentos. 
5- Organização das demandas conforme o risco

6- Organização da demanda formatação das agendas, número de atendimentos, por profissional, por período

7- Estratégia de saúde da família-PSF

8-Procedimentos clínicos básicos

\section{9- Protocolos clínicos e de regulação para encaminhamentos para atendimento de especialidades}

10- Doenças sistêmicas de alta prevalência, relacionadas diretamente com o tratamento odontológico

\section{1- Atendimento a grupos prioritários e programas}

A avaliação de risco permite que o serviço de saúde o estabeleça como critério para definição de prioridade no atendimento. Deve ser realizado por profissional da saúde que identifica os usuários que necessitam de atenção priorizada. A adoção deste critério, não significa a exclusão dos indivíduos sem atividade de doença. Os protocolos podem contribuir para a definição de prioridades, levando em consideração agravos para: doença cárie, periodontal, câncer de boca, traumatismo, edentulismo.

Oito atendimentos por período: Deve haver o Atendimento Programado caracterizase por um conjunto de ações clínicas e de promoção de saúde, ofertadas de forma planejada a grupos priorizados. Este atendimento destina-se à clientela cadastrada, do território, que deve ser priorizada através de agendamento, para realizar o tratamento odontológico necessário e deve ocupar, no mínimo, 50\% do tempo clínico. O Atendimento à Urgência visa eliminar e/ou controlar a dor de qualquer pessoa, cadastrada ou não, que demande atendimento (Eventos hemorrágicos; Eventos que exijam supressão da dor ou controle de episódio infeccioso, tais como: pulpectomias, extrações simples, pericoronarites, pericementites, alveolites, drenagem de abcesso; Eventos traumáticos como reimplantes dentários e cimentação provisória de peças protéticas) Após a finalização do atendimento de urgência, conforme o procedimento realizado, o paciente cadastrado, deve ser orientado para dar continuidade ao tratamento ou encaminhado à especialidade.

A incorporação de equipes de Saúde Bucal na estratégia tem o objetivo de ampliar o acesso da população brasileira às ações de promoção, prevenção e recuperação da saúde bucal, melhorar os indicadores de saúde, além de incentivar a reorganização desta área na atenção básica.

O protocolo deve prever procedimentos clínicos básicos, exemplo Restaurações preferencialmente provisórias e definitivas, pulpotomias; curativos endodônticos, raspagem, alisamento e polimento; procedimentos preventivos, exodontias e outros procedimentos cirúrgicos.

O protocolo deve ser também dirigido à organização dos fluxos de gerenciamento do sistema de saúde local, para referência e contra-referência da Atenção Básica para atenção especializada-CEO. Deve-se encaminhar preferencialmente, pacientes em tratamento nas Unidades Básicas de Saúde, nas Unidades de Saúde da Família ou referenciados de outros Centros de Especialidades ou Hospitais. O usuário deve ser encaminhado, por meio de formulários próprios exames complementares e radiografias após a eliminação da dor e com ações realizadas para controle da infecção bucal (adequação do meio bucal, terapia periodontal básica remoção dos focos de infecção e selamento provisório das cavidades de cárie).

O protocolo deve prever situações de pacientes com doenças sistêmicas que exijam cuidados extras durante o tratamento odontológico, tais como: diabete mellito ( $\mathrm{dm})$, gravidez, hipertensão arterial, cardiopatas, tuberculose pulmonar, hanseníase, convulsão/epilepsia

O protocolo deve visar a organização da atenção à Saúde Bucal por meio do ciclo de vida do indivíduo e conter informações e direcionamento no atendimento à grupos prioritários como gestante (programa meu bem querer), clínica do bebê, idoso. levando em consideração o tratamento diferenciado e os cuidados a serem oferecidos. 


\section{Tabela 2}

Caracterização dos profissionais da saúde participantes da Oficina de Discussão, Distrito Oeste, Ribeirão Preto-SP.

\begin{tabular}{lccccccc}
\hline Grupos & Dentistas & Enfermeiros & $\begin{array}{c}\text { Auxiliar } \\
\text { Odontológico }\end{array}$ & Usuários & $\begin{array}{c}\text { Auxiliar de } \\
\text { Enfermagem }\end{array}$ & $\begin{array}{c}\text { Agente } \\
\text { Administrativo }\end{array}$ & DACA \\
\hline Gl & 2 & - & 1 & 1 & 1 & 1 & 1 \\
G2 & 2 & 1 & 1 & 1 & - & - & 1 \\
G3 & 2 & 1 & 1 & - & 1 & - & 2 \\
\hline
\end{tabular}

DACA- Divisão de Avaliação, Auditoria e Controle da Secretaria Municipal de Saúde de Ribeirão Preto

Tabela 3

Resultado da Oficina e Validação das Categorias, Distrito Oeste, Ribeirão Preto-SP e nível de concordância segundo a Escala Likert.

\begin{tabular}{cccc}
\hline & & Grupos & \\
Nível de Concordância para cada categoria & Gl & G & G \\
\hline 1 & V & V & V \\
2 & V & IV & V \\
3 & V & V & IV \\
4 & V & V & V \\
5 & V & I & IV \\
6 & V & V & IV \\
7 & V & V & V \\
8 & V & IV & IV \\
9 & V & I & I \\
10 & V & V & V \\
11 & V & IV & II \\
\hline
\end{tabular}

\section{Resultados e Discussão}

Ao final da Oficina chegou-se a um consenso geral, conforme preconiza a técnica, com $80 \%$ de aceitação. Na Tabela 3 está descrito o resultado da Oficina com a validação das categorias no respectivo nível de concordância segundo a Escala Likert. No quadro V estão representadas e descritas as Categorias modificadas pelos grupos na Etapa três.

À semelhança do que Spinola $(1984)^{8}$ apresenta, a "Técnica Delphi deve ser construída através de um processo grupal que tem por finalidade obter, comparar e direcionar julgamento de peritos para um con- senso sobre um tópico particular, promovendo a convergência de opiniões".

Neste estudo a técnica Delphi se mostrou capaz de alcançar um alto grau de aceitação e concordância. Os itens considerados necessários e adequados que resultaram do processo servem como guia para a posterior confecção de um protocolo clínico e de regulação no município de Ribeirão Preto.

Durante o processo foi reconhecido um empenho dos sujeitos da pesquisa em gerar as listas com as sugestões, sendo verificado um elevado grau de envolvimento, na medida em que não houve questionário sem ser preenchido e também demonstraram empe- 


\section{Quadro V}

\section{Categorias modificadas pelos grupos na Etapa três}

\section{CATEGORIASMODIFICADAS}

\section{2-PROMOÇÃODA SAÚDE}

É muito importante para efetivação da Atenção Básica, pois objetiva a melhoria na qualidade de vida e a redução dos riscos à saúde, por meio da construção de políticas públicas e programas preventivos, como por exemplo: ações de fluorterapia, ações de educação em saúde, procedimentos preventivos, atendimento clínico individual na Unidades de Saúde quando (G1: sendo realizadas por todas as unidades de saúde responsáveis pela atenção básica no município) (G2:quando necessário )

\section{3-ORGANIZAÇÃO DO ACESSO (G3:e urgência)}

A ordem de chegada não deve ser o principal critério para o atendimento dos casos, mas a sua gravidade ou o sofrimento do paciente. Se prioriza o atendimento a qualquer urgência, quando esta estiver ocasionando dor ou sofrimento ao usuário. A urgência, inclusive, é um momento importante para a detecção de indivíduos com maior vulnerabilidade.

\section{5-ORGANIZAÇÃO DAS DEMANDAS CONFORME O RISCO}

A avaliação de risco como critério para definição de prioridade no atendimento. Embora priorize esta situação, não significa a exclusão dos indivíduos sem atividade de doença. Os protocolos podem contribuir para a definição de prioridades, levando em consideração agravos para (G2 e G3: para a saúde bucal)

6-ORGANIZAÇÃO DA DEMANDA - FORMATAÇÃO DAS AGENDAS, NÚMERO DE ATENDIMENTOS, POR PROFISSIONAL, POR PERÍODO

8 atendimentos por período (G1:de quatro horas.): deve haver o atendimento programado de forma planejada à clientela cadastrada, do território, que deve ser priorizada através de agendamento, para realizar o tratamento odontológico necessário. (G3: preconizando a classificação de risco e vulnerabilidade, com resolubilidade) Atendimento à Urgência visa eliminar e/ou controlar a dor de qualquer pessoa,cadastrada ou não, que demande atendimento( Eventos hemorrágicos; Eventos que exijam supressão da dor ou controle de episódio infeccioso, tais como: pulpectomias, extrações simples, pericoronarites, pericementites, alveolites, drenagem de abcesso; Eventos traumáticos como reimplantes dentários e cimentação provisória de peças protéticas.) Após a finalização do atendimento de urgência, conforme o procedimento realizado, o paciente cadastrado, deve ser orientado para dar continuidade ao tratamento ou encaminhado à especialidade.(G1:após a realização da atenção básica)

\section{8-PROCEDIMENTOS CLINICOS BÁSICOS}

O protocolo deve conter (G2 e G3: todos) procedimentos clínicos básicos.

\section{9-PROTOCOLOS CLÍNICOS E DE REGULAÇÃO PARA ENCAMINHAMENTOS PARA ATENDIMENTO DE ESPECIALIDADES}

O protocolo deve ser também dirigido à organização dos fluxos de referência e contra-referência da Atenção Básica para atenção especializada - CEO. Especialidades como: cirurgia e traumatologia buco-maxilo-facial, lesões suspeitas, traumatologia buco-maxilo-facial, cirurgia ortognática, dentística especializada, endodontia, odontopediatria, periodontia, prótese dentária, implantodontia, ortodontia, oclusão, pacientes (G3: com necessidades especiais, radiologia e hiv/aids. O usuário deve ser encaminhado com eliminação da dor e com ações realizadas para controle da infecção bucal (G2: com o cumprimento total dos procedimentos da atenção básica). O encaminhamento deverá ser feito por meio de formulários de referência/contra-referência,acompanhados ou não de exames complementares e radiografias..

\section{1-ATENDIMENTOA GRUPOS PRIORITÁRIOS EPROGRAMAS}

O protocolo deve visar a organização da atenção à Saúde Bucal por meio da faixa etária do indivíduo e conter informações e direcionamento no atendimento à grupos prioritários como gestante (programa meu bem querer), clínica do bebê, idoso. Levando em consideração o (G2: as peculiaridades de cada ciclo de vida) tratamento diferenciado e os cuidados a serem oferecidos. (G3:discriminar todos os cuidados aos grupos) 
nho e principalmente reprodução de idéias de acordo com o contexto atual da saúde.

Foi identificada a grande preocupação com relação ao acolhimento, promoção de saúde, avaliação do risco, trabalho em equipe e organização da demanda.por parte dos profissionais advindos das unidades de PSF

No entanto, houve a tendência dos sujeitos da pesquisa advindos das UBS tradicionais em valorizar ainda mais as competências práticas, técnicas, indicando ser um grupo que não foi estimulado a refletir sobre o seu compromisso social e exercício de cidadania, mas que foram preparados tecnicamente para a realização dos procedimentos odontológicos com enfoque no individuo, na esfera particular e não com o enfoque para a coletividade, na esfera pública. 9,10

Devemos dar destaque para a última etapa deste processo grupal onde a adaptação da técnica no momento da união de diferentes profissionais da saúde para a oficina de discussão na terceira etapa, trouxe "o olhar" de outros profissionais para a questão da saúde bucal e promoção de saúde e organização da demanda, dentre outros aspectos que envolvem a participação da equipe. Nela observou-se o esforço em promover o campo comum de competências, através de uma maior integração da saúde bucal aos serviços de saúde em geral, a conjugação de saberes e práticas que apontem para a promoção e vigilância em saúde, para revisão das práticas assistenciais que incorporam a abordagem familiar e a defesa da vida.

\section{Conclusőes}

O presente estudo apresenta-se como uma estratégia para implantação de novas propostas organizativas e gerenciais para unidades básicas, secundárias e terciárias, pois parte da suposição de que a reordenação do processo de trabalho em saúde deve levar em conta uma perspectiva que aponte para a criação de vínculo entre trabalhadores e usuários, para a constituição de espaços institucionais de co-gestão e o trabalho de natureza interdisicplinar.

A técnica utilizada se baseou na escuta dos indivíduos envolvidos por meio das respostas dos questionários, a fim de guiar para uma prática dos procedimentos que realmente serão realizados e com eficácia. Deste modo, as normas e consensos estarão de acordo com a realidade enfrentada pelas Unidades de Saúde.

A participação atuante dos profissionais de saúde na oficina permitiu que fossem e se sentissem "coautores" da construção do protocolo de saúde bucal e, conseqüentemente, à vontade para a efetiva implementação das práticas propostas comprovado no conteúdo do quadro II.

Por fim, coloca-se o desafio de difundir novos elementos, respeitando a cultura local e contribuindo com o "empoderamento" dos sujeitos coletivos. Tornando-os capazes de autogerirem seus processos de trabalho, com responsabilidade e compromisso, contribuindo para o desenvolvimento dos novos arranjos organizacionais que estimulam o vínculo das equipes com a produção de saúde e que lhes facilita, ao mesmo tempo, a própria realização pessoal e profissional fortalecendo a autonomia de todos os atores sociais, inclusive dos usuários no controle do processo saúdedoença-cuidado.

\section{Apoiador}

Financiamento: Fundação Waldemar Barnsley Pessoa - parte Integrante do Projeto "Avaliação da Implantação de um Conjunto de Dispositivos para Fortalecimento da Atenção Básica e do Sistema Local de Saúde"

Prêmio Prof. Dr. Guilherme Simões - $1^{\circ}$ Lugar em Pesquisa Científica do IX EPATESPO e VIII Congresso Paulista de Odontologia em Saúde Coletiva, Secretaria de Estado da Saúde e Secretaria Municipal de Saúde de Santo André.

\footnotetext{
ABSTRACT

Currently the development and use of protocols in the area of health as a tool has contributed to the Organization and standardization of clinical practice. Model of the study: this study is to estimate qualitatively. Goal of the study: this research if proposed using the Delphi Technique to achieve a consensus among the participants of the survey in the consolidation of the axles, concepts and categories that will contribute to the creation and implementation of the Protocol in dental health Public health network of the city of Ribeirão Preto. Methodology: Delphi Technique was used in the search for convergence of views. The initial sample of random character from sweepstakes between units of Health district west of Ribeirão
} 
Preto, was composed by 13 dentists and the method was described in 3 steps. Started with the Group dental units selected, which responded to a series of questions and the results of this first phase compiled and the summary of the results communicated again to members to reply again upon reconsideration of his replies. The end product was discussed in the workshop comprised dental practitioners interviewed representatives of health teams, Secretary of health and of users of the service so that the instrument could be built containing the views of all those involved in the process. Results: Showed that the technique Delphi favoured decision-making professionals and users about consolidation concepts, own the primary dental health for the development of dental health care, collaborating potentially for rational decisions and improving quality of care. Conclusion: Delphi technique proved effective instrument in seeking consensus on Dental public health.

Keywords: Clinical Protocols. Primary Health Care. Oral Health.

\section{Referências bibliográficas}

1. Brasil. Ministério da Saúde. Cadernos da Atenção Básica no 17: Saúde Bucal; 2006.

2. Ducan B, Schimidt MI, Giugliani ERJ. Medicina ambulatorial: conduta de atenção primária baseada em evidências. Prática de atenção primária. 3ㄹed. Porto Alegre: Artmed;1992

3. Gontijo LPT. Construindo as competências do cirurgião-dentista na atenção primária em saúde [Tese Doutorado]. Ribeirão Preto: Escola de Enfermagem de Ribeirão Preto - USP; 2007.

4. Prefeitura Municipal de Ribeirão Preto. Secretaria Municipal da Saúde. Plano de Saúde de Ribeirão Preto 2005 - 2008. Janise Braga Barros Ferreira (coord.). Ribeirão Preto: Secretaria Municipal da Saúde; 2005.

5. Grant JS, Kenney MR. Using the Delphi technique to examine the content vality of nursing diagnoses. Nurs Diagn. 1992; 3: $12-22$
6. Ribeirão Preto. Secretaria Municipal da Saúde. Ribeirão Preto, SP. Disponível em http://www.ribeiraopreto.sp.gov.br/ index.html. Acesso em maio de 2008.

7. Rosa WAG, Labate RC. A contribuição da saúde mental para o Programa Saúde da Família. Rev Bras enferm. 2003; 56: 230-5.

8. Spinola, A.W.P. Delpos: Proposta tecnológica alternativa. São Paulo. Faculdade de Saúde Pública. Universidade de São Paulo, 1984.

9. Starfield B. Atenção Primária: equilíbrio entre necessidades de saúde, serviços e tecnologia. Brasília: UNESCO/Ministério da Saúde; 2002.

10. Wright JTC, Giovinazzo R.A. Delphi - uma ferramenta de apoio ao planejamento prospectivo. Caderno de Pesquisa em Administração, São Paulo, 2000; 1: 52-65. 